\title{
Evaluation and significance of C-reactive protein in the clinical diagnosis of severe pneumonia
}

\author{
JIANJUN WU ${ }^{1,2^{*}}$, YU JIN ${ }^{2,3^{*}}$, HAILONG LI ${ }^{1}$, ZHIPING XIE $^{4}$, JINSONG $\mathrm{LI}^{4}$, \\ YUANYUN $\mathrm{AO}^{4}$ and ZHAOJUN DUAN ${ }^{4}$ \\ ${ }^{1}$ Gansu Traditional Chinese Medical University; ${ }^{2}$ School of Basic Medical Sciences, Lanzhou University, Lanzhou, \\ Gansu 730000; ${ }^{3}$ Nanjing Children's Hospital, Medical School of Nanjing University, Nanjing, Jiangsu 210008; \\ ${ }^{4}$ National Institute for Viral Disease Control and Prevention, China CDC, Beijing 100052, P.R. China
}

Received April 23, 2014; Accepted December 12, 2014

DOI: $10.3892 / \mathrm{etm} .2015 .2491$

\begin{abstract}
Severe pneumonia is a major cause of mortality in children. The present study evaluated the diagnostic value of serum C-reactive protein (CRP) levels for cases of severe pneumonia. A total of 862 children, hospitalized for acute respiratory tract infections, were evaluated between September 2008 and February 2011; the serum levels of CRP were measured in all the children. Bacterial identification was performed, while polymerase chain reaction was used to detect the 12 respiratory viruses. Multivariate logistic regression analysis was performed with independent [CRP, proportion of neutrophils (NEUT), body temperature, sputum production, age and dyspnea] and dependent (severe and mild pneumonia) variables for clinical diagnosis, which produced three new variables that represented an individual's predictive value: Pre-1, Pre-2 and Pre-3. A receiver operating characteristic (ROC) curve was generated using the new variables to assess their predictive value for severe pneumonia. Of the 862 patients, 108 individuals were diagnosed with severe pneumonia and 754 individuals had mild pneumonia. Increased levels of CRP were associated with severe pneumonia and bacterial infection $(\mathrm{P}<0.05)$. Multivariate logistic regression analysis found that severe pneumonia was associated with the levels of CRP, body temperature, expectoration, age, NEUT and dyspnea $(\mathrm{P}<0.05)$. The ROC curve of the regression diagnostics model sequentially presented CRP, CRP and the other five correlative variables (NEUT + body temperature + sputum production + age + dyspnea) and the other five
\end{abstract}

Correspondence to: Dr Zhaojun Duan, National Institute for Viral Disease Control and Prevention, China CDC, 100 Ying-Xin Street, Beijing 100052, P.R. China

E-mail: zhaojund@126.com

Professor Yu Jin, Nanjing Children's Hospital, Medical School of Nanjing University, 72 Guangzhou Road, Nanjing, Jiangsu 210008 , P.R. China

E-mail: jinyuldyy@163.com

${ }^{*}$ Contributed equally

Key words: C-reactive protein, severe pneumonia, clinical diagnosis correlative variables used to diagnose severe pneumonia. The area under curve values were determined as 0.550 for Pre-1 [95\% confidence interval (CI), 0.490-0.609], 0.897 for Pre-2 (95\% CI, $0.861-0.932$ ) and 0.893 for Pre-3 (95\% CI, 0.855-0.931). The results revealed that the six correlative variables had improved accuracy in the diagnosis of severe pneumonia. The serum levels of CRP were strongly associated with bacterial infection and severe pneumonia. Therefore, the CRP level, along with other parameters, may be used as early indicators of severe pneumonia development. However, the efficiency of the CRP level alone to diagnose severe pneumonia was found to be limited.

\section{Introduction}

Pneumonia is the most frequent cause of mortality in children under five years of age in a number of developing countries $(1,2)$. Community-acquired pneumonia (CAP) is an important causative mechanism of pneumonia in infants and young children in Asia and Africa. In total, $30 \%$ of CAP cases progress to severe pneumonia due to the absence of expedient diagnosis and treatment $(3,4)$. There is also evidence that the number of cases of severe pneumonia is increasing (5). Severe pneumonia is defined as requiring hospitalization in an intensive care unit (6-8); the condition frequently results in mortality.

Early and effective treatment of pneumonia is crucial. The World Health Organization (WHO) recommends standard treatments for mild and severe pneumonia; however, the lack of appropriate drugs and equipment in developing countries remains a problem $(3,8)$. Furthermore, severe pneumonia perpetuates nosocomial infection-associated mortalities $(9,10)$. Prior to pneumonia progressing to a severe state, timely diagnosis is essential to reduce the mortality rate and improve prognosis. A number of microorganisms are associated with pneumonia; however, the individual pathogens are difficult to identify. The diagnosis of mild and severe pneumonia is primarily based on clinical presentation and physical examination. (9)

C-reactive protein (CRP) is an acute-phase protein that reacts with $\mathrm{C}$ polysaccharide from Pneumococci to produce a complex (11). When tissue injury and/or acute inflammation occurs, CRP is produced by the liver in response to stimulation by interleukin- 6 and tumor necrosis factor- $\alpha$ (12). Previous studies have shown that continuous high levels of serum 
procalcitonin and CRP in the incipient stage of acute respiratory infections increases the risk of progression to a critical disease state (12-14). An integrated evaluation of the clinical symptoms and the levels of CRP may improve the predictability of the clinical course and prognosis of pneumonia (15). In patients with a high risk of pneumonia, the diagnostic value of the CRP level is unknown. Prior to the development of severe pneumonia, the significance of clinical symptoms, signs and inflammatory markers in patients with pneumonia is not clear. Thus, a large-scale study is required to confirm the diagnostic accuracy of CRP compared with the clinical symptoms and signs in terms of predicting the development of severe pneumonia. Severe pneumonia can be caused by bacterial, viral and mixed infections. The association between high serum levels of CRP and the presence of pathogenic microorganisms is also unclear. The present study investigated the significance of $\mathrm{CRP}$ in the clinical diagnosis of severe pneumonia.

\section{Materials and methods}

Patients and clinical specimens. Nasopharyngeal airway secretions (NPAs) and the clinical data from 862 children with mild and severe pneumonia, who were hospitalized at the Pediatric Treatment Center of Hunan Provincial People's Hospital (Changsha, China), were collected between September 2008 and February 2011. All the children had their serum levels of CRP measured. Clinical diagnoses were classified according to the WHO standards (16). Two clinicians established the diagnoses independently and an additional clinical expert was consulted if a consensus was not reached. Clinical data were collected from medical records. If records were incomplete, the patient's family was consulted.

Ethics statement. The study was approved by the Research Ethics Committee of Lanzhou University (Lanzhou, China) and the Institutional Review Board of the China Center for Disease Control and Prevention (Beijing, China). Specimens and clinical data were collected following the acquisition of informed consent; all participants provided written informed consent.

Bacterial culture. All NPAs were placed in a virus transport medium (containing $200 \mathrm{U} / \mathrm{ml}$ penicillin, $200 \mathrm{U} / \mathrm{ml}$ streptomycin, $200 \mathrm{U} / \mathrm{ml}$ amphotericin $\mathrm{B}$ and $0.125 \%$ bovine serum albumin) and stored at $-80^{\circ} \mathrm{C}$ until required. The specimens were inoculated on blood agar, China blue agar and Sha agar (Bianming Biotechnology Co., Ltd., Nanjing, China), and incubated for $24 \mathrm{~h}$ at $37^{\circ} \mathrm{C}$. To separate the colonies, all bacteria were identified using the bioMérieux VITEK232 automatic bacteria identification system and ATB Expression semi-automatic detector (bioMérieux, Marcy l'Etoile, France).

Detection of the CRP levels. Immunoturbidimetry was used to measure the serum levels of CRP using the Ary360 automatic rate turbidimetric system (Beckman Coulter, Inc., Brea, CA, USA), according to the manufacturer's instructions. The CRP reagents and calibration liquid were obtained from Beckman Coulter, Inc.

Detection of the 12 respiratory viruses. Viral DNA and RNA was extracted from $200 \mu 1$ NPA samples using QIAamp ${ }^{\circledR}$ viral
DNA and RNA mini-kits (Qiagen, Hilden, Germany). Reverse transcription was performed using Superscript II ${ }^{\circledR}$ Reverse Transcriptase (Invitrogen Life Technologies, Carlsbad, CA, USA) and random primers (Invitrogen Life Technologies). A routine polymerase chain reaction (PCR) assay was used to detect human bocavirus, metapneumovirus, respiratory syncytial virus, influenza virus types $A$ and $B$, parainfluenza virus types 1-3, human rhinovirus, human coronaviruses $(\mathrm{HCoV}$, NL63 and HKU1) and adenovirus, using rTaq DNA polymerase (Takara Biotechnology Co., Ltd., Dalian, China). The PCR products were resolved on a $1.5 \%$ agarose gel. The positive amplicons were purified using a QIAquick PCR Purification kit (Qiagen) and subsequently sequenced. To validate the amplification process and exclude contamination, positive and negative controls (Qiagen) were used. The positive controls contained the target gene fragment that had been isolated and purified in our laboratory; the negative controls were of the the same PCR reaction system but did not contain the viruses' target gene fragment. All laboratory tests were conducted at the National Institute for Viral Disease Control and Prevention (China Center for Disease Control and Prevention).

Statistical analysis. A t-test was used to compare the two groups if the data conformed to an approximate normal distribution, while the $\chi^{2}$ test was used to compare categorical data. Contingency table analysis was used to generate contingency table data. A multivariate logistic regression diagnosis model for the CRP levels, CRP levels + relative proportion of neutrophils in white blood cells (NEUT) + clinical symptoms and signs (body temperature, sputum production, age and dyspnea) and NEUT + clinical symptoms and signs generated three new variables with predictive values, defined as Pre-1, Pre-2 and Pre-3, respectively. Receiver operating characteristic (ROC) curves included the new test variables (Pre-1, Pre-2 and Pre-3) alongside the clinical diagnosis. The clinical diagnosis was the dependent variable, which was defined as follows: Severe pneumonia, 1; mild pneumonia, 0 . Independent variables were defined as follows: CRP level (measured values); NEUT (measured values); body temperature (measured values); sputum production (yes, 1 ; no, 0); age (months), dyspnea (yes, 1; no, 0); bacterial infection (yes, 1; no, 0); viral infection (yes, 1, no, 0); cough (yes, 1, no, 0); and gender (male, 1, female, 2). All statistical analyses were performed using SPSS 13.0 software (SPSS, Inc., Chicago, IL, USA), and $\mathrm{P}<0.05$ was considered statistically significant.

\section{Results}

Patient information. The male to female ratio was $1.55: 1$ and the ratio of mild to severe pneumonia cases was $6.84: 1$. The average patient age was $19.18 \pm 9.145$ months. Positive cases (CRP level of $\geq 10 \mathrm{mg} / \mathrm{l}$ ) of severe pneumonia accounted for $25.92 \%(28 / 108)$ of the total cases, while positive cases with mild pneumonia (CRP level of $<10 \mathrm{mg} / \mathrm{l}$ ) accounted for $16.18 \%$ of the total cases $(122 / 754 ; \mathrm{P}<0.05)$. The average body temperature of those with severe pneumonia was $38.53 \pm 1.03^{\circ} \mathrm{C}$ and $38.29 \pm 1.10^{\circ} \mathrm{C}$ in those with mild pneumonia $(\mathrm{t}=-2.16 ; \mathrm{P}=0.03)$. The relative proportion of neutrophils in patients with severe pneumonia was $44.49 \pm 19.71 \%$, compared with $38.46 \pm 21.28 \%$ for those with mild pneumonia $(\mathrm{P}<0.05)$. The frequency of 
Table I. Viral and bacterial detection status in patients with severe and mild pneumonia.

\begin{tabular}{lll}
\hline Classification & Severe pneumonia & Mild pneumonia \\
\hline None detected, $\%(\mathrm{n})$ & & 0.12 \\
$\quad$ Virus & $39.81(43 / 108)$ & $47.88(361 / 754)$ \\
Bacteria & $37.04(40 / 108)$ & $49.47(373 / 754)$ \\
Detection of a single species, \% (n) & & $39.39(297 / 754)$ \\
$\quad$ Virus & $44.44(48 / 108)$ & $10.88(82 / 754)$ \\
Bacteria & $17.59(19 / 108)$ & 0.02 \\
Detection of two or more species, $\%(\mathrm{n})$ & & $12.73(96 / 754)$ \\
Virus & $15.74(17 / 108)$ & $39.67(299 / 754)$ \\
Bacteria & $45.37(49 / 108)$ & 0.23 \\
Coinfection, $\%(\mathrm{n})$ & & $28.91(218 / 754)$ \\
Yes & $39.81(43 / 108)$ & $71.09(536 / 754)$ \\
No & $60.19(65 / 108)$ & 0.02 \\
\hline
\end{tabular}

Table II. Contingency table analysis of CRP and associated variables.

\begin{tabular}{lccc}
\hline & \multicolumn{2}{c}{ CRP $(\mathrm{mg} / \mathrm{l})$} & \\
\cline { 2 - 3 } Associated variables & $>10.0$ & $\leq 10.0$ & R-value \\
\hline Severe pneumonia & 28 & 80 & -0.081 \\
Yes & 122 & 630 & 0.02 \\
No & & & -0.086 \\
Bacterial infection & 67 & 383 & 0.02 \\
Positive & 89 & 323 & 0.010 \\
Negative & & & 0.77 \\
Viral infection & 54 & 267 & \\
Positive & 87 & 454 & \\
Negative & & & \\
\hline
\end{tabular}

CRP, C-reactive protein.

sputum production in patients with severe pneumonia was $47.22 \%$ (51/108), compared with 58.36\% (440/754) in individuals with mild pneumonia $(\mathrm{P}<0.05)$. The percentage of individuals with dyspnea in the severe pneumonia group was 9.26\% (10/108), while $14.19 \%$ (107/754; P>0.14) individuals were affected in the mild pneumonia group.

Viral and bacterial detection. In $60.19 \%$ (65/108) of patients with severe pneumonia, at least one virus was detected, compared with $51.99 \%(392 / 754)$ of patients with mild pneumonia. This difference was not statistically significant $\left(\chi^{2}=2.47 ; \mathrm{P}=0.12\right)$. At least one bacterial species was detected in $62.96 \%(68 / 108)$ of the severe pneumonia cases, compared with $50.53 \%(381 / 754)$ of the mild pneumonia cases; this difference was statistically significant $(\mathrm{P}=0.02$; Table I).

Relevance of CRP and associated variables. Serum levels of CRP were higher in the severe pneumonia group when compared with the mild pneumonia group $(\mathrm{P}<0.05$; independent sample t-test). An increased level of CRP was shown to be strongly associated with severe pneumonia $(\mathrm{r}=-0.081 ; \mathrm{P}<0.05)$ and bacterial infection $(\mathrm{r}=-0.086 ; \mathrm{P}<0.05)$. However, increased levels of CRP had no association with viral infection $(\mathrm{P}>0.05$; Table II). If bacterial infection was considered the gold standard in the diagnosis of pneumonia, the diagnostic value of CRP would be as follows: a sensitivity of $13.39 \%$ with a false negative rate of $86.61 \%$ and a specificity of $76.20 \%$ with a false positive rate of $23.80 \%$.

Logistic regression model of severe pneumonia. With the severity of pneumonia as the dependent variable, the CRP level, body temperature, expectoration, bacterial infection, viral infection, cough, gender, age, NEUT and dyspnea were used as covariates. Using multivariate logistic regression analysis, a preliminary regression model was established, and the covariates of bacterial infection, viral infection, cough and gender were excluded $(\mathrm{P}>0.1)$. The regression model was assessed using a likelihood-ratio test and the goodness of fit of the regression model was demonstrated to be satisfactory $(\mathrm{P}<0.01$; Table III). The regression model was as follows: 
Table III. Multivariate logistic regression analysis of severe pneumonia.

\begin{tabular}{|c|c|c|c|c|c|c|c|}
\hline \multirow{2}{*}{$\begin{array}{l}\text { Concomitant } \\
\text { variable }\end{array}$} & \multirow{2}{*}{$\begin{array}{l}\text { Regression } \\
\text { coefficient }\end{array}$} & \multirow[b]{2}{*}{ SE } & \multirow[b]{2}{*}{ Wald } & \multirow[b]{2}{*}{ P-value } & \multirow[b]{2}{*}{ OR } & \multicolumn{2}{|c|}{$95 \% \mathrm{CI}$} \\
\hline & & & & & & Lower & Upper \\
\hline $\operatorname{CRP}\left(\mathrm{X}_{1}\right)$ & 0.011 & 0.006 & 3.344 & 0.067 & 1.011 & 0.999 & 1.024 \\
\hline Age $\left(\mathrm{X}_{2}\right)$ & -0.038 & 0.011 & 12.158 & 0.000 & 0.962 & 0.942 & 0.983 \\
\hline Body temperature $\left(\mathrm{X}_{3}\right)$ & 0.325 & 0.134 & 5.824 & 0.016 & 1.383 & 1.063 & 1.801 \\
\hline Expectoration $\left(\mathrm{X}_{4}\right)$ & -0.853 & 0.283 & 9.108 & 0.003 & 0.426 & 0.245 & 0.742 \\
\hline Dyspnea $\left(\mathrm{X}_{5}\right)$ & 3.360 & 0.286 & 137.936 & 0.000 & 28.801 & 16.438 & 50.461 \\
\hline $\operatorname{NEUT}\left(\mathrm{X}_{6}\right)$ & 0.005 & 0.003 & 2.324 & 0.127 & 1.005 & 0.999 & 1.011 \\
\hline Constant & -14.780 & 5.150 & 8.237 & 0.004 & 0.000 & & \\
\hline
\end{tabular}

CRP, C-reactive protein; NEUT, proportion of neutrophils; AUC, area under curve; SE, standard error; OR, odds ratio; CI, confidence interval.

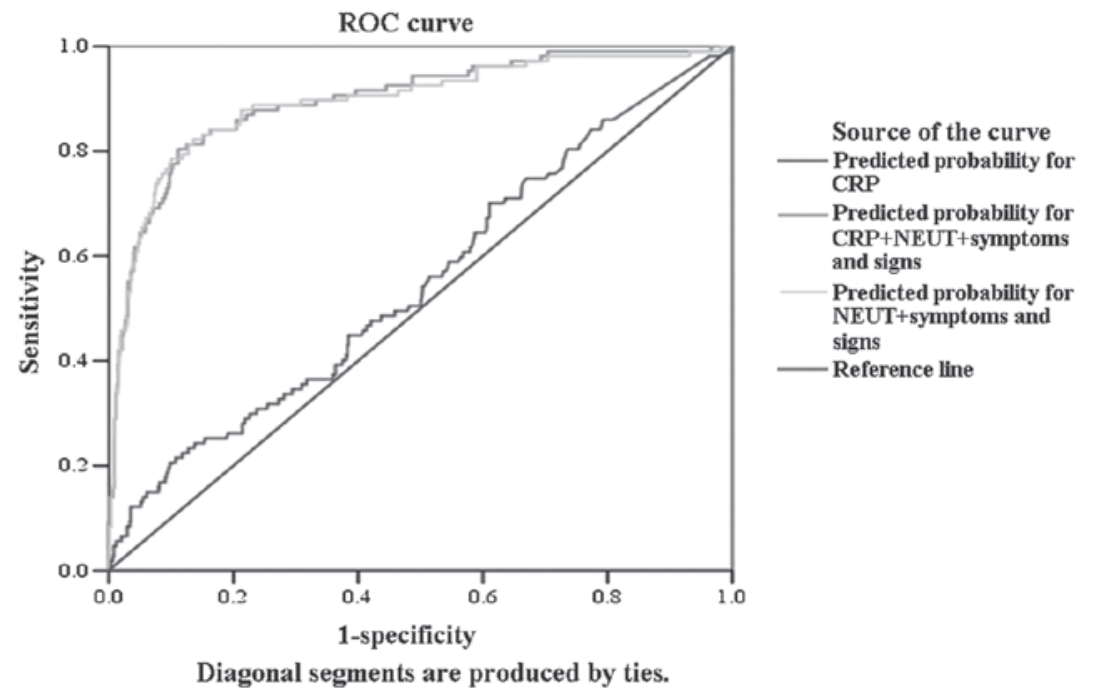

Figure 1. ROC curve for Pre-1 in the diagnosis of severe pneumonia. CRP, C-reactive protein; NEUT, proportion of neutrophils; ROC, receiver operator characteristic.

Table IV. AUC values, generated using regression diagnostics, for Pre-1, Pre-2 and Pre-3.

\begin{tabular}{lccccc}
\hline & & & & \multicolumn{2}{c}{$95 \%$ CI } \\
\cline { 5 - 6 } Diagnostic & AUC & SE & P-value & Lower & Upper \\
\hline Pre-1 & 0.550 & 0.030 & 0.096 & 0.490 & 0.609 \\
Pre-2 & 0.897 & 0.018 & 0.000 & 0.861 & 0.932 \\
Pre-3 & 0.893 & 0.019 & 0.000 & 0.855 & 0.931 \\
\hline
\end{tabular}

AUC, area under curve; SE, standard error; CI, confidence interval.

$$
\begin{aligned}
\operatorname{Logit}(P)=-14.067 & +0.012 X_{1}+0.283 X_{2}+0.408 X_{3}-0.071 X_{4} \\
& +0.008 X_{5}+0.655 X_{6}
\end{aligned}
$$

Where $\mathrm{X}_{1}$ was the CRP level, $\mathrm{X}_{2}$ was the age; $\mathrm{X}_{3}$ was the body temperature; $X_{4}$ was expectoration; $X_{5}$ was dyspnea; and $X_{6}$ was NEUT.
Diagnostic value of the clinical factors and ROC curve analysis for severe pneumonia cases. The area under the curve (AUC) of a clinical diagnostic model was generated and included the CRP level (Pre-1), CRP level + NEUT + symptoms and signs (Pre-2), and the severe pneumonia clinical diagnosis. The ROC plot was calculated to evaluate the predictive value and function. The model was also evaluated by constructing a ROC plot. The model was optimal using Pre-2, with an AUC of 0.897 [95\% confidence interval (CI), 0.861-0.932], followed by Pre-3 (AUC, 0.893; 95\% CI, 0.855-0.931) and Pre-1 (AUC, 0.550; 95\% CI, 0.490-0.609; Fig. 1; Table IV).

\section{Discussion}

Economic, social and living conditions have greatly improved in middle- and low-income countries over the past two decades, which has resulted in a significant reduction in the incidence rate of CAP. However, the morbidity and mortality rates of CAP remain high in the majority of developing countries $(2,3)$, with severe pneumonia the leading cause of mortality in cases of CAP. However, the treatment of severe pneumonia remains 
poor in developing countries; thus, early diagnosis is crucial. The standard method for CAP diagnosis is the detection of a new infiltrate on chest radiographs, in addition to recently acquired respiratory signs and symptoms (17-19). In primary care, physicians rely solely on patient history and a physical examination (20). Clinical practice has demonstrated that the evaluation of clinical signs and symptoms alone is not sufficient for the diagnosis of CAP (21). To predict the development of severe pneumonia, a number of factors should be considered.

According to the WHO-recommended treatment plan for preventing mortality from severe pneumonia (19-21), special measures, including supportive care, appropriate immunotherapy and intensive care treatment, should be administered in a timely manner. The NEUT and levels of CRP are inflammatory biomarkers, which improve the predictive power and diagnostic value for the risk assessment of severe pneumonia (21-23). Previous studies addressed the diagnostic significance of CRP in relation to CAP (24-27), while additional studies indicated that CRP was associated with disease severity and mortality in hospitalized patients with CAP $(28,29)$. However, the association between CRP and severe pneumonia has not, to the best of our knowledge, been reported. The present study demonstrated that serum levels of CRP and NEUT were elevated in patients with severe and mild pneumonia. The results demonstrated that the proportion of cases with severe pneumonia (CRP level $\geq 10 \mathrm{mg} / \mathrm{l}$ ) was higher compared with the mild pneumonia group, indicating that the persistent elevation of serum CRP levels may be associated with the condition of the patient and the development of severe pneumonia. As shown in the current study, the clinical symptoms and signs, levels of CRP alone and the NEUT had poor diagnostic accuracy in terms of predicting severe pneumonia.

Bacterial infections (Pneumococcus, hemolytic streptococci and Haemophilus spp.), viral infections (respiratory syncytial virus, influenza virus, human rhinoviruses and bocavirus) and mixed infections are major causes of pneumonia, and particularly of severe pneumonia (30-32). Antibiotic therapy for severe pneumonia based on pathogen separation and detection is rare, and the presence of a non-infectious differential diagnosis is usually suspected only after the failure of antibiotic therapy. This leads to risks associated with a lack of treatment, and potentially life-threatening, non-bacterial diseases. Serum procalcitonin levels may be used to predict the probability of a bacterial infection in patients with severe chronic obstructive pulmonary disease and pneumonia (33). Notably, the present study found that high levels of serum CRP were strongly associated with the detection of bacteria. The use of serum CRP levels as a diagnostic indicator of bacterial infection resulted in a false negative rate of $86.61 \%$ and a specificity of $76.20 \%$; the serum levels of CRP were higher in the severe pneumonia patients compared with the mild pneumonia group. However, the data obtained in the present study indicated that viral detection rates did not significantly differ between the severe and mild pneumonia groups. By contrast, a previous study found that viruses were commonly detected in children with severe pneumonia in the Philippines (32).

The diagnosis of severe pneumonia in children from a number of developing countries is limited by the lack of $\mathrm{X}$-ray facilities. In addition, the diagnosis relies primarily on clinical symptoms (34). A diagnosis based on clinical suspi- cion and traditional clinical experience tends to delay therapy, resulting in severe consequences $(3,20,35)$. The most important risk factors for treatment failure are a longer duration of therapy, overprescription of antibiotics and non-compliance with WHO recommendations (36). Thus, a number of challenges remain for the early detection of severe pneumonia. The current guidelines for diagnosis are based on clinical signs and symptoms, which makes a rapid diagnosis in children extremely important for a positive clinical outcome. CRP levels alone should not be relied upon for a pneumonia diagnosis and clinical symptoms should be considered. Using univariate and multivariate logistic regression analyses, the present study demonstrated that significant factors for the prediction of severe pneumonia were the NEUT, body temperature, sputum production, age and dyspnea. The ROC curve using the new variables that were generated by multivariate regression analysis and the diagnostic gold standard for severe pneumonia demonstrated the reliability of these factors to diagnose severe pneumonia. According to the data, the likelihood of developing severe pneumonia was higher in younger patients with elevated levels of CRP, an increased NEUT, high fever, a frequent cough and dyspnea. Patients with these risk factors should receive timely and appropriate treatment. In the current study, the AUC of Pre-2 was 0.897 (95\% CI, 0.861-0.932), which indicates that the regression model containing Pre-2 has improved diagnostic accuracy for severe pneumonia. In addition, this result indicates the diagnostic value of using a combination of indicators. When only the levels of CRP are considered, as opposed to the composite variables, the diagnostic value and significance of the levels of CRP were limited. However, measuring the CRP levels may assist in the diagnosis and treatment of CAP by physicians in clinical practice. The high morbidity and mortality rates of severe pneumonia attract worldwide attention. To reduce the mortality rate, awareness of the importance of early detection of pneumonia should be increased, along with measures to prevent disease development.

\section{Acknowledgements}

The study was partly supported by grants from the '973' National Key Basic Research Program of China (no. 2007CB310500), the Key Program of Nanjing Medical Science and Technique Development Foundation (no. ZKX09008) and the Medical Science and Technique Development Foundation of Jiangsu Province Health Department (no. H200949).

\section{References}

1. Liu L, Johnson HL, Cousens S, et al: Global, regional, and national causes of child mortality: an updated systematic analysis for 2010 with time trends since 2000. Lancet 379: 2151-2161, 2012.

2. Bryce J, Boschi-Pinto C, Shibuya K, Black RE; WHO Child Health Epidemilogy Reference Group: WHO estimates of the causes of death in children. Lancet 365: 1147-1152, 2005.

3. Rudan I, O'Brien KL, Nair H, et al: Epidemiology and etiology of childhood pneumonia in 2010: estimates of incidence, severe morbidity, mortality, underlying risk factors and causative pathogens for 192 countries. J Glob Health 3: 10401, 2013.

4. Dekate PS, Mathew JL, Jayashree M and Singhi SC: Acute community acquired pneumonia in emergency room. Indian J Pediatr 78: 1127-1135, 2011. 
5. Aydoğdu M, Ozyilmaz E, Aksoy H, Gursel G and Ekim N Mortality prediction in community-acquired pneumonia requiring mechanical ventilation; values of pneumonia and intensive care unit severity scores. Tuberk Toraks 58: 25-34, 2010.

6. Mandell LA, Wunderink RG, Anzueto A, et al; Infectious Diseases Society of America; American Thoracic Society: Infectious Diseases Society of America/American Thoracic Society consensus guidelines on the management of community-acquired pneumonia in adults. Clin Infect Dis 44 (Suppl 2): S27-S72, 2007.

7. Lim WS, van der Eerden MM, Laing R, et al: Defining community acquired pneumonia severity on presentation to hospital: an international derivation and validation study. Thorax 58: 377-382, 2003.

8. Ewig S, de Roux A, Bauer T, et al: Validation of predictive rules and indices of severity for community acquired pneumonia Thorax 59: 421-427, 2004.

9. Zar HJ, Madhi SA, Aston SJ and Gordon SB: Pneumonia in low and middle income countries: progress and challenges. Thorax 68: 1052-1056, 2013.

10. Soofi S, Ahmed S, Fox MP, et al: Effectiveness of community case management of severe pneumonia with oral amoxicillin in children aged 2-59 months in Matiari district, rural Pakistan: a cluster-randomised controlled trial. Lancet 379: 729-737, 2012

11. Póvoa P: C-reactive protein: a valuable marker of sepsis. Intensive Care Med 28: 235-243, 2002.

12. Paiva MB, Botoni FA, Teixeira AL Jr, et al: The behavior and diagnostic utility of procalcitonin and five other inflammatory molecules in critically ill patients with respiratory distress and suspected 2009 influenza a H1N1 infection. Clinics (Sao Paulo) 67: 327-334, 2012.

13. Medeiros IL, Terra RM, Choi EM, Pego-Fernandes PM and Jatene FB: Evaluation of serial C-reactive protein measurements after surgical treatment of pleural empyema. Clinics (Sao Paulo) 67: 243-247, 2012

14. Suberviola B, Castellanos-Ortega A, Llorca J, Ortiz F, Iglesias D and Prieto B: Prognostic value of proadrenomedullin in severe sepsis and septic shock patients with community-acquired pneumonia. Swiss Med Wkly 142: w13542, 2012.

15. van Vugt SF, Broekhuizen BD, Lammens C, et al; GRACE consortium: Use of serum $\mathrm{C}$ reactive protein and procalcitonin concentrations in addition to symptoms and signs to predict pneumonia in patients presenting to primary care with acute cough: diagnostic study. BMJ 346: f2450, 2013.

16. Heffelfinger JD, Davis TE, Gebrian B, Bordeau R, Schwartz B and Dowell SF: Evaluation of children with recurrent pneumonia diagnosed by World Health Organization criteria. Pediatr Infect Dis J 21: 108-112, 2002

17. Niederman MS, Mandell LA, Anzueto A, et al: Guidelines for the management of adults with community-acquired pneumonia Diagnosis, assessment of severity, antimicrobial therapy, and prevention. Am J Respir Crit Care Med 163: 1730-1754, 2001.

18. Mandell LA, Bartlett JG, Dowell SF, File TM Jr, Musher DM and Whitney C; Infections Diseases Society of America: Update of practice guidelines for the management of community-acquired pneumonia in immunocompetent adults. Clin Infect Dis 37 $1405-1433,2003$

19. Dalhoff K: Worldwide guidelines for respiratory tract infections: community-acquired pneumonia. Int J Antimicrob Agents 18 (Suppl 1): S39-S44, 2001.

20. Goossens H and Little P: Community acquired pneumonia in primary care. BMJ 332: 1045-1046, 2006.
21. Muller B, Harbarth S, Stolz D, et al: Diagnostic and prognostic accuracy of clinical and laboratory parameters in community-acquired pneumonia. BMC Infect Dis 7: 10, 2007.

22. Chalmers JD, Mandal P, Singanayagam A, et al: Severity assessment tools to guide ICU admission in community-acquired pneumonia: systematic review and meta-analysis. Intensive Care Med 37: 1409-1420, 2011

23. Carratala J, Fernandez-Sabe N, Ortega L, et al: Outpatient care compared with hospitalization for community-acquired pneumonia: a randomized trial in low-risk patients. Ann Intern Med 142: 165-172, 2005.

24. File TM Jr, Lode H, Kurz H, Kozak R, Xie H and Berkowitz E; 600 Study Group: Double-blind, randomized study of the efficacy and safety of oral pharmacokinetically enhanced amoxicillin-clavulanate $(2,000 / 125$ milligrams $)$ versus those of amoxicillin-clavulanate (875/125 milligrams), both given twice daily for 7 days, in treatment of bacterial community-acquired pneumonia in adults. Antimicrob Agents Chemother 48: 3323-3331, 2004

25. Zha Y, Yang X, Yuan J, et al: The effect of continuous renal replacement therapy on the outcome of severe pneumonia in patients receiving long-term immunosuppressants. Zhonghua Jie $\mathrm{He} \mathrm{He} \mathrm{Hu} \mathrm{Xi} \mathrm{Za} \mathrm{Zhi} \mathrm{36:} \mathrm{169-172,} 2013$ (In Chinese).

26. Diez-Padrisa N, Bassat Q, Morais L, et al: Procalcitonin and C-reactive protein as predictors of blood culture positivity among hospitalised children with severe pneumonia in Mozambique. Trop Med Int Health 17: 1100-1107, 2012.

27. Kolditz M, Höffken G, Martas P, et al; CAPNETZ Study Group: Serum cortisol predicts death and critical disease independently of CRB-65 score in community-acquired pneumonia: a prospective observational cohort study. BMC Infect Dis 13: 90 , 2012.

28. Menendez R, Martinez R, Reyes S, et al: Biomarkers improve mortality prediction by prognostic scales in community-acquired pneumonia. Thorax 64: 587-591, 2009.

29. Lee JH, Kim J, Kim K, et al: Albumin and C-reactive protein have prognostic significance in patients with community-acquired pneumonia. J Crit Care 26: 287-294, 2011

30. Feikin DR, Njenga MK, Bigogo G, et al: Viral and bacterial causes of severe acute respiratory illness among children aged less than 5 years in a high malaria prevalence area of western Kenya, 2007-2010. Pediatr Infect Dis J 32: e14-e19, 2013.

31. Turner C, Turner P, Carrara V, et al: High rates of pneumonia in children under two years of age in a South East Asian refugee population. PLoS One 8: e54026, 2013.

32. Suzuki A, Lupisan S, Furuse Y, et al: Respiratory viruses from hospitalized children with severe pneumonia in the Philippines. BMC Infect Dis 12: 267, 2012.

33. Daubin C, Parienti JJ, Fradin S, et al: Procalcitonin levels and bacterial aetiology among COPD patients admitted to the ICU with severe pneumonia: a prospective cohort study. BMC Infect Dis 9: 157, 2009.

34. Schwarz NG, Sarpong N, Hünger F, et al: Systemic bacteraemia in children presenting with clinical pneumonia and the impact of non-typhoid salmonella (NTS). BMC Infect Dis 10: 319, 2010.

35. Fischer JE, Bachmann LM and Jaeschke R: A readers' guide to the interpretation of diagnostic test properties: clinical example of sepsis. Intensive Care Med 29: 1043-1051, 2003.

36. Pakistan Muticentre Amoxycillin Short Course Therapy (MASCOT) Pneumonia Study Group: Clinical efficacy of 3 days versus 5 days of oral amoxicillin for treatment of childhood pneumonia: a multicentre double-blind trial. Lancet 360: $835-841,2002$ 